\title{
The challenges of venoarterial extracorporeal membrane oxygenation for postcardiotomy cardiogenic shock
}

\author{
Charlesworth Michael $^{1}$ (D) $\cdot$ Rajamiyer Venkateswaran ${ }^{2}$
}

Received: 22 June 2020 / Revised: 22 September 2020 / Accepted: 25 September 2020 / Published online: 7 November 2020

(C) Indian Association of Cardiovascular-Thoracic Surgeons 2020

\begin{abstract}
Postcardiotomy cardiogenic shock describes the syndrome of refractory cardiac performance following cardiac surgery. The use of venoarterial extracorporeal membrane oxygenation (VA-ECMO) for the management of postcardiotomy cardiogenic shock is controversial, and there are at least three scenarios where it may be necessary: first, pre-emptive postoperative VA-ECMO, where the decision for postoperative mechanical support is made prior to surgery, for example, in the context of poor pre-operative cardiac function; second, early yet unplanned post-cardiopulmonary bypass VA-ECMO following a long duration of cardiopulmonary bypass due to, for example, unexpected surgical complications; third, late rescue VA-ECMO following several attempts at weaning, either immediately following cardiopulmonary bypass or following transfer to the intensive care unit. The use of mechanical circulatory support for postcardiotomy cardiogenic shock is further complicated by the wide range of available devices, the availability of VA-ECMO in different centres, variations in experience and expertise as a function of local VAECMO workload, and regional variations in the diagnosis and management of postcardiotomy cardiogenic shock. Furthermore, survival appears to be low for such patients and it is not yet possible to predict who will survive. Many questions remain, however, such as those in relation to practices around patient selection, how best to study long-term outcomes, the ethics and efficacy of ECMO in such patients, and on all aspects of clinical decision-making. This review sets these clinical challenges in the context of the available evidence, including that from our centre.
\end{abstract}

Keywords Venoarterial extracorporeal membrane oxygenation $\cdot$ Postcardiotomy $\cdot$ Cardiogenic shock $\cdot$ Cardiac surgery $\cdot$ ECMO

\section{Introduction}

Technology is nothing. What's important is that you have a faith in people, that they're basically good and smart, and if you give them tools, they'll do wonderful things with them. It's not the tools that you have faith in - tools are just tools. They work, or they don't work. It's people you have faith in or not.

Steve Jobs, 1994

For most cardiac surgical procedures, separation from cardiopulmonary bypass (CPB) is uneventful with the patient

Charlesworth Michael

michael.charlesworth@mft.nhs.uk

1 Department of Cardiothoracic Critical Care, Anaesthesia and ECMO, Wythenshawe Hospital, Manchester, UK

2 Department of Cardiothoracic Surgery and Transplantation, Wythenshawe Hospital, Manchester, UK subsequently able to support their own circulatory demands. For some, vasopressors, inotropes and intra-aortic balloon pump (IABP) counterpulsation are required. Rarely $(\sim 0.7-$ $3.6 \%$ ) cardiac performance following surgery is refractory to this support and there is a need therefore to consider peripheral or central venoarterial extracorporeal membrane oxygenation (VA-ECMO) as a bridge to recovery, ventricular assist device (VAD) implantation or transplantation. Postcardiotomy cardiogenic shock (PCCS) describes the syndrome of refractory cardiac performance following cardiac surgery, yet there is no agreed consensus with regard to how it should be defined or managed. This is further complicated by the wide range of devices available to treat PCCS; the availability of such interventions in different centres; variations in experience and expertise as a function of local VA-ECMO workload and regional variations in the diagnosis and management of PCCS. This article considers the use of VA-ECMO to treat non-transplant PCCS through our own experiences at Wythenshawe Hospital, Manchester, UK. 


\section{History and development}

Despite a recent upsurge in clinical activity, ECMO is by no means a new technology having been developed in the $1950 \mathrm{~s}$ primarily for CPB [1]. The desire to provide longer periods of mechanical circulatory support than can be offered with CPB gave rise to a simpler, closed circuit configuration, which we now recognise as ECMO. It was not until the 1970s however that the first clinical report of veno-venous extra corporeal membrane oxygenation for acute respiratory distress syndrome (ARDS) emerged in the literature [2]. Despite initial promise, interest faded following reports of high mortality for ARDS treated with veno-venous extra corporeal membrane oxygenation (VV-ECMO) as compared with traditional medical management [3]. Thereafter, ECMO research and practice were confined to the fringes of paediatric practice until a recent upsurge in adult interest in the 1990s. It is likely that this second wave of adult ECMO practice was born out of technological improvements in catheters, circuits, pumps and oxygenators in combination with general improvements in critical care medicine. Even more recently, the practice of VV-ECMO for respiratory failure has seen significant advances through acquired expertise and experience during the 2009 H1N1 influenza [4] and COVID-19 pandemics [5]. Concurrently, several randomised controlled trials (RCTs) and observational studies of VV-ECMO for respiratory failure in adults have reported good outcomes as compared with traditional medical management $[6,7]$. Despite this and notwithstanding continued reporting of successes from across the world, it has not yet been possible to pool such data in a meta-analysis to provide an overall appraisal of VV-ECMO due to considerable data heterogeneity [8]. In the UK, it is funded as a centrally commissioned service and equipoise has been lost. The efficacy of postcardiotomy VA-ECMO is even more so difficult to prove through traditional research methods despite observational data suggesting that it provides a significant survival benefit for what is ordinarily a condition with an extremely high mortality [9].

\section{Approaches and techniques}

When VA-ECMO is initiated in theatre following intrathoracic CPB, the 'central' configuration is usually selected, as cannulation sites are identical. The sternum can be left open to enable re-exploration for bleeding. Peripheral VA-ECMO may be selected for cases where extrathoracic CPB is used, for example repeat surgery, minimally invasive surgery and cases where there is disease of the great vessels, such as a thoracic aortic dissection. Additionally, peripheral VAECMO or extracorporeal cardiopulmonary resuscitation (ECPR) may be initiated in the intensive care unit without the need for re-sternotomy. Combined central and peripheral venous drainage (VVA-ECMO) may also be employed to enhance the venous drainage capabilities of the circuit.
Despite their similarities, VA-ECMO has several advantages over CPB in the context of PCCS. Firstly, it allows mechanical circulatory support for days or weeks, as compared with hours. It is a closed circuit with shorter tubing, no venous reservoir and less stagnation of flow in the cardiac and pulmonary vasculature. It therefore requires lower doses of intravenous unfractionated heparin. Although there is always a risk of intrathoracic or intracerebral haemorrhage, this risk is lessened though the reduced need for systematic anticoagulation. In addition to enabling closure of the sternum through the tunnelling of tubing, VA-ECMO achieves a more physiological haematocrit and blood flow, more efficient delivery of oxygen to tissues and normothermia. It allows concurrent cardiac ejection and thus further reduces the risk of thrombus formation. Finally, patients receiving VA-ECMO can be cared for in the critical care environment whereas those undergoing CPB cannot.

Of course, whilst any comparison of $\mathrm{CPB}$ and VAECMO gives an insight into their technical differences, pragmatically, the clinical choice is in fact between VAECMO or no circulatory support beyond IABP counterpulsation and vasopressors/inotropes (Fig. 1). Regardless of the underlying cause, PCCS without mechanical circulatory support has an extremely poor prognosis and most cases will not survive the immediate postoperative course. VA-ECMO allows the opportunity for the acutely shocked heart to recover without also having to meet the demands of the circulation.

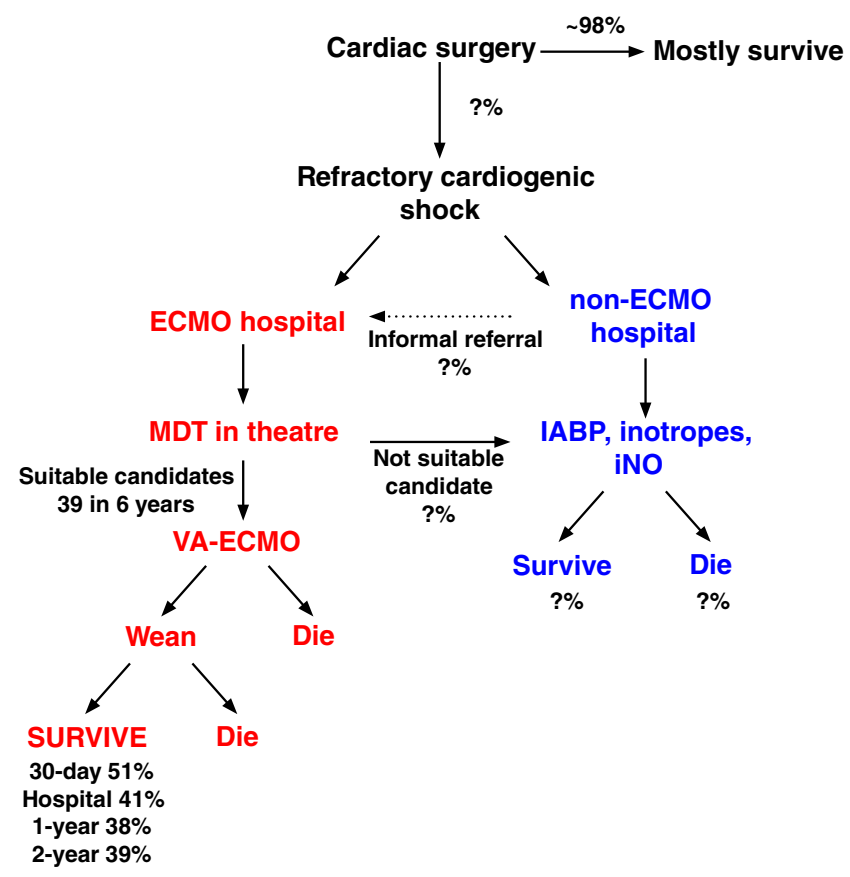

Fig. 1 A flow diagram of the referral pathway for VA-ECMO for PCCS at our centre. One of the key aims for the future is to determine the missing data marked by a '?'. MDT, multidisciplinary team; IABP, intra-aortic balloon pump; iNO, inhaled nitric oxide 
Despite these advantages, VA-ECMO for PCCS is an expensive resource with an as yet unproven evidence base, and with funding bodies such as National Institute for Clinical Excellence (NICE) uncertain with regard to its safety profile [10]. Highly skilled staff are required at all stages of the process, from initiation in theatre or critical care to maintenance, weaning and rehabilitation thereafter. Individual hospitals providing ECMO absorb the financial cost of their VA service, as there is currently no central funding in the UK. Such centres are usually expected to participate in the decision-making process for postcardiotomy VA-ECMO initiation at non-ECMO centres and sometimes even retrieve patients from other centres. There are controversies around the use of VA-ECMO for PCCS in nontransplant or low-volume ECMO centres in the UK, as whilst it is possible to initiate VA-ECMO in such centres, patients are arguably best cared for in high-volume ECMO centres [11]. The financial and logistical arrangements for such patients are therefore complex and decisions are taken on a case-by-case basis. Some typical initial targets are provided below for reference only; however, most targets are also selected on a case-by-case basis.

- Flow of $60-80 \mathrm{~mL} / \mathrm{kg} / \mathrm{min}$

- $\mathrm{FiO}_{2}$ of 1.0

- $\mathrm{SpO}_{2}$ of $95-100 \%$

- mean arterial pressure (MAP) of 60-90 mmHg

- $\mathrm{pH}$ of 7.35-7.45

- Haematocrit of greater than $28 \%$

- Functioning platelet count of greater than 80

- activated clotting time (ACT) 180-220 s

Anticoagulation practices vary between centres, clinicians and patients. The extracorporeal life support organisation (ELSO) have attempted to standardise this through their recent guideline [12]. For PCCS VA-ECMO, patients may not require an initial dose of unfractionated heparin (UFH). In the absence of bleeding and when the ACT falls into the target range, a heparin infusion of 20-50 units $/ \mathrm{kg} / \mathrm{h}$ can be used for maintenance. A reduced platelet count, increased urine output or concurrent renal replacement therapy may increase the need for UFH [13]. Heparin induced thrombocytopenia and heparin resistance lie outside the scope of this article.

Weaning from VA-ECMO can be as a bridge to recovery, VAD implantation or transplantation. Where recovery is anticipated, signs of ejection, pulsatility and contraction should be seen using transoesophageal echocardiography (TOE) in at least the first week. TOE should also rule out significant valvular pathology and estimate ventricular function. ECMO flow rates can be incrementally reduced concurrently with TOE examination. If appropriate, the ECMO circuit is clamped and a trial of recirculation is initiated for $1-4 \mathrm{~h}$. The cannulae are continuously flushed during this period with $\mathrm{UFH} /$ saline to avoid cannula thrombosis. Decannulation is considered if haemodynamic parameters allow. Where VA-
ECMO is a bridge to VAD implantation, this is considered only following the resolution of end-organ dysfunction.

\section{Indications and patient selection}

The technical aspects of VA-ECMO for PCCS are arguably more explicit than the tacit subtleties of decision-making, timing and patient selection. Whilst two recent systematic reviews conclude a significant survival benefit of VAECMO over and above alternative strategies, the majority of included research is retrospective and observational, with no randomised or controlled trials [14, 15]. Retrospective observational studies provide a good means for centres to report their results, but those who wish to make predictions for future patients must interpret this with great caution. Most are in fact reporting a case series and are therefore inherently prone to selection and information biases, amongst others [9, 15]. Due to these problems, the majority of postcardiotomy VA-ECMO research has been unable to implicate prognostic indicators that can be used by clinicians to make evidence-based decisions. At our institution therefore, VA-ECMO for PCCS can only be initiated on a case-by-case basis with the agreement of four consultants including two surgeons and two anaesthetists with adequate clinical ECMO experience. The indication for VA-ECMO for PCCS is simple - inadequate tissue perfusion as evidenced by hypotension and low cardiac output despite adequate intravascular volume that is refractory to inotropes, vasopressors and IABP counterpulsation. In general, all planned operative procedures should be complete and the chances of successful recovery should be high. Deciding where VA-ECMO is contraindicated is more complex. Where cardiac failure is considered irreversible in combination with a patient that is not a candidate for VAD implantation or heart transplantation, VA-ECMO for PCCS is absolutely contraindicated. Relative contraindications include advanced age, chronic organ dysfunction, multiple co-morbidities, established multi-organ failure syndrome (MOFS), obesity and when it is not possible to administer anticoagulants.

There is a dissimilarity between heart transplant and nonheart transplant patients with regard to this decision-making process. In our own experience, the threshold for postoperative VA-ECMO is somewhat lower following heart and/or lung transplantation as compared with non-transplant procedures. It is often used pre-emptively, but it may also be used for primary graft dysfunction, acute organ rejection or refractory cardiogenic shock. A discussion of postoperative VAECMO following heart and/or lung transplantation lies outside the scope of this article, but its increasing role for such cases is increasing VA-ECMO expertise at regional transplant centres. We have never bridged a patient to long-term mechanical circulatory support or transplantation following VA-ECMO for non-transplant PCCS. 
Table 1 Several key studies reporting outcomes for patients treated with VA-ECMO for PCCS. Our own results from Manchester are highlighted at the bottom of the table

\begin{tabular}{|c|c|c|c|c|c|c|}
\hline & $\begin{array}{l}\text { Number of } \\
\text { patients }(n)\end{array}$ & $\begin{array}{l}\text { 30-day survival } \\
(\%)\end{array}$ & $\begin{array}{l}\text { Hospital discharge } \\
\text { survival }(\%)\end{array}$ & $\begin{array}{l}\text { 1-year survival } \\
(\%)\end{array}$ & $\begin{array}{l}\text { 2-year survival } \\
(\%)\end{array}$ & Comment \\
\hline $\begin{array}{l}\text { Distelmaier et al. } 2016 \\
\quad[17]\end{array}$ & 385 & - & $56 \%$ & $40 \%$ & - & Included transplant and ECPR patients \\
\hline Fux et al. 2018 [18] & 105 & - & $44 \%$ & - & - & $\begin{array}{l}\text { The most comprehensive analysis in } \\
\text { the literature }\end{array}$ \\
\hline Liden et al. 2009 [19] & 33 & - & $45 \%$ & $36 \%$ & - & Included heart transplant patients \\
\hline Loforte et al. 2014 [20] & 155 & - & $46 \%$ & - & - & $\begin{array}{l}\text { High rate of peripheral VA-ECMO } \\
\quad(52.5 \%)\end{array}$ \\
\hline Luo et al. 2009 [21] & 36 & - & $42 \%$ & - & - & Low mean age (49 years) \\
\hline Mikus et al. 2013 [22] & 14 & - & $43 \%$ & $35 \%$ & - & Only 14 patients \\
\hline Wang et al. 2009 [23] & 62 & - & $55 \%$ & $52 \%$ & - & Included heart transplant patients \\
\hline Yang et al. 2014 [24] & 12 & - & $67 \%$ & - & - & Only 12 patients \\
\hline $\begin{array}{l}\text { Charlesworth et al. } \\
2019 \text { [25] }\end{array}$ & 39 & $51.3 \%$ & $41 \%$ & $37.5 \%$ & $38.5 \%$ & $\begin{array}{l}\text { No cannula- or ECMO-related } \\
\text { complications }\end{array}$ \\
\hline
\end{tabular}

\section{Clinical outcomes}

The generic complications of VA-ECMO are accepted to be thrombosis (1-22\%), haemorrhage (5-79\%), limb ischaemia (13-25\%), infection (17-49\%) and irreversible neurological sequelae (10-33\%) [16]. A summary of several key studies reporting outcomes for patients undergoing VA-ECMO for PCCS is provided below (Table 1). This does not represent a systematic synthesis of all the relevant literature and studies are presented to provide a representative overview only.

Most studies are single-centre retrospective analyses where survival data (usually in the form of hospital discharge) and complication rates are reported. Many also report the cause of death or try to establish a relationship between survival and factors such as serum lactate, vasopressor use/dose, age, risk stratification scores (EuroSCORE), surgery type, urgency, duration of support and obesity. Pragmatically, it would be unwise to pool the above clinical studies with all other related studies due to considerable data heterogeneity. Retrospective non-randomised non-controlled data of this type is unlikely to reflect the true efficacy of VA-ECMO for PCCS or the true incidence of procedure-related complications. These limitations together with the possibility of high complication rates are a major barrier in the way of funding at present. For 39 patients receiving VA-ECMO for PCCS at our centre, the operations undertaken were coronary artery bypass grafts (CABG) $(10.3 \%)$; aortic valve replacement $+/-$ CABG (28.2\%); mitral valve surgery +/- CABG (15.4\%); double/ triple valve surgery $+/-$ CABG $(20.5 \%)$; pericardectomy (5.1\%); and thoracic aortic surgery (20\%). The median (IQR [range]) ECMO run was 6 (4-9 [1-35]) days [25].

Unfortunately, the answer is not to simply perform a randomised controlled trial (RCT) [9]. This would invoke the unethical randomisation of patients to a non-VA-ECMO arm that will most likely lead to death as compared with a treatment that is arguably beyond experimental and where outcomes are reasonable [25]. Furthermore, we argue that to statistically treat VA-ECMO as a binary intervention with an on/off switch, such as a pharmaceutical agent, is a gross oversimplification. It is a complex multi-variable technology applied to a heterogeneous patient population at the extremes of pathology and physiology. Practices also vary between clinicians and between centres. Even if several RCTs were to be conducted, there may still be considerable data heterogeneity that will preclude meta-analyses. Therefore, clinical decisionmaking is determined by consensus, debate, summing factors for and against, negotiation and wider contextual factors such as location and resources.

\section{Conclusion}

The questions of who should provide VA-ECMO for PCCS or which patients are most likely to benefit from it have no easy answers. It is also unlikely that traditional research will provide a breakthrough solution to guide clinicians anytime soon [9]. What is known is that PCCS without VA-ECMO is fatal for the majority of cases. It is likely that transplant centres are more familiar with postcardiotomy VA-ECMO as it is commonly employed pre-emptively following heart and/or lung transplantation. Due to this familiarity together with increased experience and expertise, it is also likely that such centres will be more proactive at an earlier stage and prior to an established MOFS. Outcomes are likely to be worse when initiated later in the PCCS course in the context of an intractable lactic/ metabolic acidosis, a MOFS and a failing heart in the critical care unit. VA-ECMO should be seen as a bundle of care and as an adjunct to other resuscitative measures as opposed to a 
binary intervention. It is likely that other factors such as the quality of medical and nursing care contribute significantly despite our inability to measure these. As outcomes appear to be better at transplant centres, there are many difficult ethical questions with regard to current arrangements for PCCS VA-ECMO in the UK. Based on our own experience together with emerging observational evidence from around the world, we argue that VA-ECMO for PCCS is far beyond experimental and that it greatly enhances cardiac surgical patient safety. We must increase collaboration to bring uniformity to practices, strive to better understand patient selection and establish an optimal bundle of care for such patients.

Funding No funding.

\section{Compliance with ethical standards}

Informed consent Not applicable being a review article.

Ethics Comm approval Not applicable being a review article.

Conflict of interest The authors declare that they have no conflict of interest.

Human and animal rights Not applicable being a review article.

\section{References}

1. Gibbon JH. Application of a mechanical heart and lung apparatus to cardiac surgery. Minn Med. 1954;37:171-85.

2. Hill JD, O'Brien TG, Murray JJ, et al. Prolonged extracorporeal oxygenation for acute post-traumatic respiratory failure (shock-lung syndrome). Use of the Bramson membrane lung. N Engl J Med. 1972;286:629-34.

3. Zapol WM, Snider MT, Hill JD, et al. Extracorporeal membrane oxygenation in severe acute respiratory failure. A randomized prospective study. JAMA. 1979;242:2193-6.

4. DeLaney E, Smith MJ, Harvey BT, et al. Extracorporeal life support for pandemic influenza: the role of extracorporeal membrane oxygenation in pandemic management. J Extra Corpor Technol. 2010;42:268-80.

5. Zochios V, Brodie D, Charlesworth M, Parhar KK. Delivering extracorporeal membrane oxygenation for patients with COVID-19: what, who, when and how? Anaesthesia. 2020;75:997-1001.

6. Peek GJ, Mugford M, Tiruvoipati R, et al. Efficacy and economic assessment of conventional ventilatory support versus extracorporeal membrane oxygenation for severe adult respiratory failure (CESAR): a multicentre randomised controlled trial. Lancet. 2009;374:1351-63.

7. Schmidt M, Hodgson C, Combes A. Extracorporeal gas exchange for acute respiratory failure in adult patients: a systematic review. Crit Care. 2015;19:99.

8. Tramm R, Ilic D, Davies AR, Pellegrino VA, Romero L, Hodgson C. Extracorporeal membrane oxygenation for critically ill adults. Cochrane Database Syst Rev. 2015:CD010381.
9. Charlesworth M, Venkateswaran R, Feddy L. When traditional research fails - the case for veno-arterial ECMO in postcardiotomy cardiogenic shock. Anaesthesia. 2017;72:1425-6.

10. Extracorporeal membrane oxygenation (ECMO) for acute heart failure in adults | Guidance and guidelines | National Institute for Clinical Excellence (NICE). https://www.nice.org.uk/guidance/ ipg482 (accessed August 16, 2020).

11. Barbaro RP, Odetola FO, Kidwell KM, et al. Association of hospitallevel volume of extracorporeal membrane oxygenation cases and mortality. Analysis of the Extracorporeal Life Support Organization Registry. Am J Respir Crit Care Med. 2015;191:894-901.

12. Extracorporeal Life Support Organisation (ELSO) Anticoagulation guideline 2014. https://www.elso.org/Portals/0/Files/ elsoanticoagulationguideline8-2014-table-contents.pdf (accessed 15 Aug 2020).

13. Chen H, Yu R-G, Yin N-N, Zhou J-X. Combination of extracorporeal membrane oxygenation and continuous renal replacement therapy in critically ill patients: a systematic review. Crit Care. 2014;18:675.

14. Khorsandi M, Dougherty S, Bouamra O, et al. Extra-corporeal membrane oxygenation for refractory cardiogenic shock after adult cardiac surgery: a systematic review and meta-analysis. J Cardiothorac Surg. 2017;12:55.

15. Charlesworth M, Venkateswaran R, Barnard J, Barker J, Feddy L. Unplanned extracorporeal life support (ECLS) following cardiac surgery: a systematic review. Anaesthesia. 2017;72:1-88.

16. Lafç G, Budak AB, Yener AÜ, Cicek OF. Use of extracorporeal membrane oxygenation in adults. Heart Lung Circ 2014; 23: 10-23.

17. Distelmaier K, Schrutka L, Binder C, et al. Cardiac arrest does not affect survival in post-operative cardiovascular surgery patients undergoing extracorporeal membrane oxygenation. Resuscitation. 2016;104:24-7.

18. Fux T, Holm M, Corbascio M, Lund LH, van der Linden J. Venoarterial extracorporeal membrane oxygenation for postcardiotomy shock: risk factors for mortality. J Thorac Cardiovasc Surg. 2018;156:1894-1902.e3.

19. Liden H, Wiklund L, Haraldsson A, Berglin E, Hultman J, Dellgren G. Temporary circulatory support with extra corporeal membrane oxygenation in adults with refractory cardiogenic shock. Scand Cardiovasc J. 2009;43:226-32.

20. Loforte A, Marinelli G, Musumeci F, et al. Extracorporeal membrane oxygenation support in refractory cardiogenic shock: treatment strategies and analysis of risk factors. Artif Organs. 2014;38: E129-41.

21. Luo X, Wang W, Hu S, et al. Extracorporeal membrane oxygenation for treatment of cardiac failure in adult patients. Interact Cardiovasc Thorac Surg. 2009;9:296-300.

22. Mikus E, Tripodi A, Calvi S, Giglio MD, Cavallucci A, Lamarra M. CentriMag venoarterial extracorporeal membrane oxygenation support as treatment for patients with refractory postcardiotomy cardiogenic shock. ASAIO J. 2013;59:18-23.

23. Wang J, Han J, Jia Y, et al. Early and intermediate results of rescue extracorporeal membrane oxygenation in adult cardiogenic shock. Ann Thorac Surg. 2009;88:1897-903.

24. Yang F, Jia Z, Xing J, et al. Effects of intra-aortic balloon pump on cerebral blood flow during peripheral venoarterial extracorporeal membrane oxygenation support. J Transl Med. 2014;12:106.

25. Charlesworth M, Garcia M, Head L, et al. Venoarterial extracorporeal membrane oxygenation for postcardiotomy cardiogenic shocka six-year service evaluation. Artif Organs. 2020;44:709-16.

Publisher's note Springer Nature remains neutral with regard to jurisdictional claims in published maps and institutional affiliations. 